\title{
Physiological Regulation of the $\beta$-Amyloid Precursor Protein Signaling Domain by c-Jun N-Terminal Kinase JNK3 during Neuronal Differentiation
}

\author{
W. Taylor Kimberly, ${ }^{1 *}$ Jessica B. Zheng, ${ }^{1 \star}$ Terrence Town, ${ }^{2}$ Richard A. Flavell, ${ }^{2}$ and Dennis J. Selkoe ${ }^{1}$ \\ ${ }^{1}$ Center for Neurologic Diseases, Harvard Medical School and Brigham and Women's Hospital, Boston, Massachusetts 02115, and ${ }^{2}$ Section of \\ Immunobiology, Howard Hughes Medical Institute, Yale University School of Medicine, New Haven, Connecticut 06520
}

\begin{abstract}
$\beta$-Amyloid precursor protein (APP) is a conserved and ubiquitous transmembrane glycoprotein strongly implicated in the pathogenesis of Alzheimer's disease but whose normal biological function is unknown. Analogy to the Notch protein suggests that APP is a cell-surface receptor that signals via sequential proteolytic cleavages that release its intracellular domain (AICD) to the nucleus. Because these cleavages are major targets for therapeutic inhibition, it is critical to elucidate their physiological function. AICD is stabilized by Fe65, interacts with the transcriptional factor Tip60, and translocates to the nucleus. Here, we show that endogenous AICD in primary neurons is detectable only during a short period of time during differentiation in culture. During this transient rise, a portion of AICD localizes to the nucleus. Subsequently, phosphorylation of the APP cytoplasmic domain at threonine 668 appears to disrupt the stabilizing interaction with Fe65 and thus downregulate AICD-mediated signaling. Furthermore, we find that the neuron-specific c-Jun N-terminal kinase JNK3, but not JNK1 or JNK2, mediates a substantial portion of this phosphorylation. We conclude that endogenous AICD undergoes tight temporal regulation during the differentiation of neurons and is negatively regulated by JNK3 via phosphorylation of APP at Thr668.
\end{abstract}

Key words: amyloid precursor protein; AICD; JNK; Alzheimer's disease; phosphorylation; signaling mechanism

\section{Introduction}

Regulated intramembrane proteolysis (RIP) is a recently discovered signaling paradigm (Brown et al., 2000) that has potential relevance for the normal function of the $\beta$-amyloid precursor protein (APP). Originally implicated in the pathogenesis of Alzheimer's disease (Selkoe, 1991), APP is a single-pass transmembrane protein without a defined function. The analogy to other RIP substrates such as Notch (Selkoe and Kopan, 2003) suggests that APP might also be a cell-surface receptor that binds a cognate ligand and signals via proteolytic release of its intracellular domain (ICD). The findings that the APP ICD (AICD) can translocate to the nucleus with Fe65 (Cao and Sudhof, 2001; Cupers et al., 2001; Kimberly et al., 2001) and that the complex can drive transcription of heterologous reporter constructs (Cao and Sudhof, 2001) further support this hypothesis.

The processing of Notch and APP by the RIP mechanism is

Received June 19, 2004; revised April 26, 2005; accepted May 1, 2005.

T.T. is supported by a Ruth L. Kirschstein National Institutes of Health/National Research Service Award/National Institute on Aging postdoctoral fellowship. R.A.F. is an investigator of the Howard Hughes Medical Institute. D.J.S. is supported by the National Institutes of Health and a Pioneer Award from the Alzheimer's Association. We thank S. Vasquez for expert assistance in preparing primary neuronal cultures. We are indebted to D. Walsh and W. Farris for helpful discussions and suggestions. We thank L.-H. Tsai for the $\alpha$ P-T668 antibody, J. Buxbaum for the Fe65 antibody, D. M. McLoughlin for the Fe65 plasmid, and J. Woodgett for the SAPK $\alpha$-p54, SAPK $\beta$-p 54 , and SAPK $\gamma$-p 46 plasmids.

*W.T.K. and J.B.Z. contributed equally to this work.

Correspondence should be addressed to Dennis J. Selkoe, Center for Neurologic Diseases, Harvard Institutes of Medicine, Room 730, 77 Avenue Louis Pasteur, Boston, MA 02115. E-mail: dselkoe@rics.bwh.harvard.edu. DOI:10.1523/JNEUROSCI.4883-04.2005

Copyright $\odot 2005$ Society for Neuroscience $\quad$ 0270-6474/05/255533-11\$15.00/0 remarkably similar. Both proteins undergo a disintegrin metalloprotease (ADAM)-mediated cleavage (also referred to as $\alpha$-secretase cleavage) that sheds most of their ectodomains. The subsequent $\mathrm{C}$-terminal fragments are cleaved by presenilin $/ \gamma$ secretase within the membrane to release the respective ICDs. The Notch ICD interacts with the CSL (CBF1, suppressor of hairless, Lag-1) proteins and translocates to the nucleus, in which it affects downstream transcriptional targets (Artavanis-Tsakonas et al., 1999). In an analogous manner, the AICD fragment can combine with Fe65 and enter the nucleus. However, in all previous studies, analysis of AICD has required exogenous overexpression of this highly labile protein. Moreover, in APPoverexpressing stable cell lines, the level of AICD appears constant, without evidence for the modulation that one might expect of a signaling system (W. T. Kimberly, J. B. Zheng, and D. J. Selkoe, unpublished data). To gain an understanding of endogenous AICD function, we focused on primary neurons. We chose this cell model because, among different organisms from Drosophila to human, neurons are the strongest expressers of APP. The neuron is also a cell type that exhibits phenotypic abnormalities when APP is either ablated (Dawson et al., 1999; Torroja et al., 1999) or overexpressed (Mucke et al., 1994; Torroja et al., 1999). Finally, the neuron is the cell type that is the most relevant to the mechanism of Alzheimer's disease. For these reasons, we hypothesized that detecting and analyzing endogenous AICD in the unique cellular environment of the neuron might be critical for elucidating its function in RIP-mediated signaling.

Here we report that endogenous neuronal AICD undergoes a 
temporally restricted upregulation over an otherwise very low baseline level. It accumulates to its highest amounts in neurons at 6 and $7 \mathrm{~d}$ in vitro (DIV) and rapidly diminishes thereafter. This spike in level is associated with nuclear localization of a fraction of AICD, suggesting that the fragment may play a role in specific nuclear transcriptional events. Interestingly, the subsequent decrease in AICD levels correlates with a marked increase in the neuron-specific phosphorylation of the precursor of AICD, the APP C-terminal fragments (CTFs). This occurs at threonine 668 (T668), an amino acid whose phosphorylation state has been shown to mediate phenotypic changes in the neurites of pheochromocytoma cell line PC12 cells (Ando et al., 1999). T668 phosphorylation has also been reported to diminish APP binding to Fe65 (Ando et al., 2001), and we now show that this change destabilizes AICD. Finally, we demonstrate that the neuronspecific c-Jun N-terminal kinase JNK3 [also called SAPK $\beta$-p54 (for review, see Davis, 2000)] is the principal kinase responsible for phosphorylation of APP at T668 in neurons. Together, our data lead to a model in which AICD signaling occurs endogenously in neurons and the phosphorylation of T668 by JNK3 negatively regulates this signaling. The brief window of increased AICD levels during neuronal differentiation raises the possibility that AICD signaling may play a role in the formation of synapses, a hypothesis consistent with both APP transgenic and knock-out animal studies (Mucke et al., 1994; Dawson et al., 1999; Torroja et al., 1999).

\section{Materials and Methods}

Plasmids and transfections. A cDNA containing an initiating methionine and the last 49 residues of the APP C terminus (C50) was amplified by PCR and inserted into the vector pcDNA3.1 $(+)$ containing the zeocin resistance gene (Invitrogen, Carlsbad, CA). The sequence was confirmed by DNA sequencing. Point mutations changing the APP Thr668 residue to Ala or Glu were performed using PCR, in which the primers contained the appropriate point mutations. For transfections, plasmids $(10 \mu \mathrm{g})$ were introduced into COS7 cells at $80-95 \%$ confluence in $10 \mathrm{~cm}$ dishes using Lipofectamine 2000 (Invitrogen) according to the instructions of the manufacturer. COS cells were left in the liposome-DNA mixture for $\sim 36 \mathrm{~h}$ before collection and analysis. Plasmids encoding wild-type human $\mathrm{APP}_{695}$ and Fe65 have been characterized previously (Kimberly et al., 2001). The plasmids encoding SAPK $\alpha$-p54 (JNK2), SAPK $\beta$-p54 (JNK3), and SAPK $\gamma$-p46 (JNK1) were a kind gift from J. Woodgett (Ontario Cancer Institute, Toronto, Ontario, Canada). All experiments were repeated at least five times to confirm reproducibility.

Cells. COS cells were maintained in standard DMEM supplemented with $10 \%$ fetal bovine serum plus penicillin and streptomycin (Invitrogen). The Chinese hamster ovary $(\mathrm{CHO})$ cells stably expressing $\mathrm{APP}_{751}$, $\mathrm{APP}_{751}$ containing V717F Alzheimer's disease-causing mutation, and $\mathrm{APP}_{695}$ have all been described previously (Koo and Squazzo, 1994; Podlisny et al., 1995). Primary mouse neurons were plated at a density of $18,000 \mathrm{cells} / \mathrm{cm}^{2}$ on poly-D-lysine-coated $10 \mathrm{~cm}$ dishes. Primary rat neurons were plated at a density of 30,000 cells $/ \mathrm{cm}^{2}$ on poly-D-lysine-coated $10 \mathrm{~cm}$ dishes. Both species of neurons were maintained in Neurobasal medium supplemented with B27 (Invitrogen), and two-thirds of the medium volume was changed on a regular schedule every 3-4 d.

Antibodies. Polyclonal antibody $\mathrm{C} 8$ to $\mathrm{APP}_{732-751}\left(\mathrm{APP}_{751}\right.$ numbering) was described previously (Haass et al., 1992). Polyclonal antibody C9 was raised to the same peptide epitope as $\mathrm{C} 8$ and was used for some experiments. For immunoprecipitations (IPs), it was used at 1:200; for Western blotting, it was used at 1:2000. The characterization and preparation of the phosphorylation-specific threonine 668 APP ( $\alpha$ P-T668) antibody was described previously (Lee et al., 2003), and this was used at 1:1000 dilution. Additional antibodies were directed to the following: ADAM10 (1:1000; Chemicon, Temecula, CA), tumor necrosis factor- $\alpha$-converting enzyme (TACE) (1:1000; Chemicon), $\beta$-amyloid precursor proteincleaving enzyme (BACE1) (Ab-2, 1:1000; Oncogene, Cambridge, MA),
Fe65 (1:1000; gift from J. Buxbaum, Mount Sinai School of Medicine, New York, NY), insulin-degrading enzyme (IDE) [IDE-1, 1:1000 (Farris et al., 2003)], JNK3 (1:1000; Upstate Biotechnology, Lake Placid, NY), phosphorylated JNK (1:1000; Cell Signaling Technology, Beverly, MA), and 12CA5 (1:1000; Roche, Basel, Switzerland).

Nuclear fractionation. Three $10 \mathrm{~cm}$ dishes of neurons cultured from the same embryonic brain batch were prepared at each stated time point for biochemical fractionation into nuclear, cytoplasmic, and membrane fractions, as described previously (Kimberly et al., 2001). After this separation, the membrane and nuclear pellets were first dissolved in $100 \mu \mathrm{l}$ of $1 \%$ SDS lysis buffer containing $50 \mathrm{~mm}$ Tris, pH 7.6, $150 \mathrm{~mm} \mathrm{NaCl}, 2$ mм EDTA, 1\% SDS, 2 mм 1,10-phenanthroline, and a protease inhibitor mixture [ $5 \mu \mathrm{g} / \mathrm{ml}$ leupeptin, $5 \mu \mathrm{g} / \mathrm{ml}$ aprotinin, $2 \mu \mathrm{g} / \mathrm{ml}$ pepstatin A, and $1 \mathrm{~mm}$ pefabloc (Roche)]. After sonication, the samples were then diluted $1: 9$ with a detergent buffer identical to the $1 \%$ SDS lysis buffer, except that the SDS was replaced with 1\% NP-40 (Fisher, Hampton, NH). For the cytoplasmic fraction, $1 \mathrm{ml}$ of this was adjusted to contain equivalent amounts of detergent as the membrane and nuclear fractions. These fractions were then subjected to C8 immunoprecipitation.

Immunoprecipitation and Western blotting. Neurons were solubilized in $150 \mu \mathrm{l}$ of sample buffer (20\% glycerol, $4 \%$ SDS, and $10 \%$ $\beta$-mercaptoethanol) containing $2 \mathrm{~mm} 1,10$-phenanthroline and boiled at $100^{\circ} \mathrm{C}$ for $5 \mathrm{~min}$. Samples used for IP were lysed in 1\% SDS lysis buffer and then sonicated at $4^{\circ} \mathrm{C}$ for $15 \mathrm{~s}$, followed by dilution in the above lysis buffer containing 1\% NP-40 substituted for SDS and phosphatase inhibitors I and II (Sigma, St. Louis, MO). Samples were immunoprecipitated with C8 polyclonal APP antibody (1:200) and $50 \mu \mathrm{l}$ of protein A-Sepharose (Sigma) for $>2 \mathrm{~h}$ at $4^{\circ} \mathrm{C}$. Immunoprecipitates were washed three times for $15 \mathrm{~min}$ each with the lysis buffer above containing $0.2 \%$ $\mathrm{NP}-40$. Samples were eluted in sample buffer at $100^{\circ} \mathrm{C}$ for $5 \mathrm{~min}$, resolved by $10-20 \%$ Tris-tricine SDS-PAGE [Invitrogen or Bio-Rad (Hercules, CA)], and transferred to $0.2 \mu \mathrm{M}$ optitran nitrocellulose (Schleicher \& Schuell, Dassel, Germany). Western blotting using the appropriate antibodies and ECL Plus detection was performed according to the instructions of the manufacturer (Amersham Biosciences, Arlington Heights, IL).

Dephosphorylation analysis. Neurons used for dephosphorylation were lysed in 1\% NP-40 lysis buffer (identical to 1\% SDS lysis buffer except that NP-40 detergent was used in lieu of SDS). Lysates were adjusted to contain $1 \times \lambda$-protein phosphatase $\left(\gamma\right.$-PP) buffer and $1 \times \mathrm{MnCl}_{2}$. One microliter of double distilled $\mathrm{H}_{2} \mathrm{O}$ or $\lambda$-PP was added to the appropriate reactions and incubated at $37^{\circ} \mathrm{C}$ for $2 \mathrm{~h}$. Samples were then analyzed by Western blotting as delineated above.

Densitometry. For densitometric analysis in the primary neurons, blots containing lysates from an equal number of neurons were probed for full-length APP, APP CTFs, AICD, ADAM10, TACE, BACE1, IDE, and Fe65. Densitometric values were analyzed using AlphaImager software (Alpha Innotech, San Leandro, CA). The same blots were stripped, reprobed with glyceraldehyde-3-phosphate dehydrogenase (GAPDH), and assessed via densitometry, and the resultant values were used to normalize each of the above proteins. No significant difference was observed with or without GAPDH normalization, confirming the accuracy of our lysate loading. Control samples in serial twofold dilutions on the same film were used to demonstrate the density at which the imaging analysis software was in the linear range.

Mouse brain analysis. Mice were anesthetized, and brains were perfused with ice-cold PBS, harvested, and immediately homogenized or placed on dry ice. Hemibrains were weighed and Dounce homogenized at $0.1 \mathrm{~g} / \mathrm{ml}$ in the SDS lysis buffer with phosphatase inhibitors I and II (Sigma). Protein $(30 \mu \mathrm{g})$ was loaded in each lane, and Western blotting was performed as above. Densitometry on these samples was performed as above, with $n=6$ separate brains for wild type and knock-out.

\section{Results}

We and others have shown previously that the AICD-like cytoplasmic fragment of APP, C60, can be stabilized by Fe65 and translocate to the nucleus (Cao and Sudhof, 2001; Cupers et al., 2001; Kimberly et al., 2001; Walsh et al., 2003). Several putative functions have been attributed to this 60 -residue recombinant 

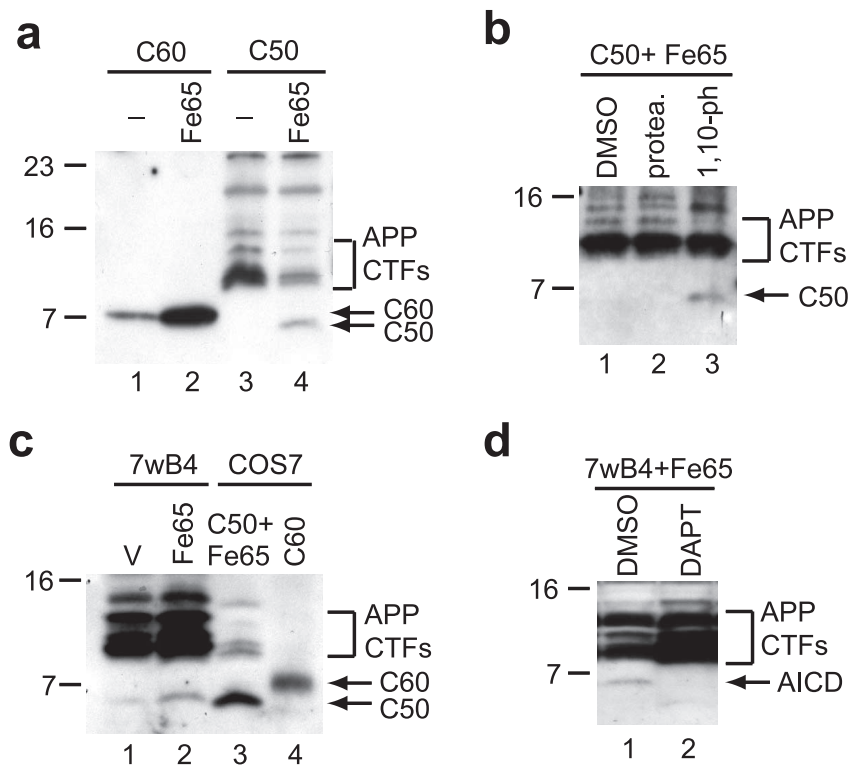

Figure 1. $\quad(50$ is more analogous to endogenous AICD than C60. $\boldsymbol{a}$, Comparison of size and Fe65-mediated stabilization of $\mathrm{C} 60$ and C50. Lanes 1 and 2 are consistent with previous experiments showing that coexpression of $\mathrm{C} 60$ with $\mathrm{Fe} 65$ results in additional stabilization (Cupers et al., 2001; Kimberly et al., 2001). (50 is similarly stabilized by Fe65, but its steady-state level is substantially lower (lanes 3,4). Identical COS7 cultures were transiently transfected with the indicated constructs. Five times the amount of protein was loaded in lanes 3 and 4 than in lanes 1 and 2, allowing detection in the latter lanes of the endogenous COS APP CTFs (bracket) and some nonspecific bands, all of which were also visible during overexposure of lanes 1 and 2 (data not shown). $\boldsymbol{b}$, The effect of protease inhibitors on C50. COS7 cells were transfected with the indicated constructs and lysed in 1\% SDS lysis buffer containing complete protease inhibitors (Roche) and the indicated additional inhibitor or solvent. The proteasome inhibitor mixture (protea.) contained $10 \mu \mathrm{M}$ MG132, $10 \mu \mathrm{M}$ lactacystin, and $10 \mu \mathrm{M}$ LLnL. 1,10-Phenanthroline (1,10-ph) was shown previously to stabilize AICD in an in vitro membrane reaction (Edbauer et al., 2002). Longer exposures revealed faint $\mathrm{C} 50$ bands in lanes 1 and 2. C, C50, but not C60, comigrates with endogenous AICD from a stable $\mathrm{CHO}$ cell line overexpressing wild-type $\mathrm{APP}_{751}$ (7wB4). Fe65 stabilizes the endogenous AICD (lane 2), which comigrates with C50. V, Vector.d, Endogenous AICD formation is prevented by a $\gamma$-secretase inhibitor. 7wB4 cells were transfected with Fe65 and then treated with the $\gamma$-secretase inhibitor DAPT (or DMSO) for $4 \mathrm{~h}$.

cytoplasmic APP fragment (Gao and Pimplikar, 2001; Roncarati et al., 2002). However, it has been found that the $\mathrm{N}$ terminus of endogenous AICD is actually 10 residues $\mathrm{C}$ terminal to the start of C60 (Gu et al., 2001; Sastre et al., 2001). To verify our previous findings with C60 (Kimberly et al., 2001), we performed similar experiments with a recombinant C50 construct that very closely mimics endogenous AICD (differing by only one residue because of the requirement for an initiating methionine in C50). Identical dishes of COS7 cells transfected with the respective plasmids demonstrated that the C50 protein is substantially less stable than C60 (Fig. 1a, compare lanes 1-2 with 3-4). Gel loading of five times more C50 protein (lane 3) than was used for C60 (lane 1) still did not yield reliable detection of $\mathrm{C} 50$ by itself. However, coexpression with Fe65 resulted in the stabilization of C50 protein (lane 4) in a manner resembling that seen with C60 (lane 2). C50 invariably migrated slightly faster than C60, as expected (Edbauer et al., 2002).

Because a previous report showed that the metalloprotease inhibitor 1,10-phenanthroline prevents the degradation of AICD generated by an in vitro membrane incubation reaction (Edbauer et al., 2002), we evaluated the stability of C50 in the presence of this compound compared with a mixture of proteasome inhibitors [MG132, lactacystin, and N-acetyl-L-leucinyl-L-leucinyl-Lnorleucinal (LLnL)]. The C50 protein was stabilized when cells were lysed in the presence of $2 \mathrm{~mm} \mathrm{1,10-phenanthroline} \mathrm{(Fig.} \mathrm{1b,}$ lane 3 ). In contrast, the proteasome inhibitor mixture had no discernible effect on detection of C50 (lane 2), consistent with previous reports (Cupers et al., 2001). We next verified that C50 comigrated with AICD generated endogenously from full-length human APP that was stably expressed in a $\mathrm{CHO}$ cell line called 7wB4. Whereas C50 comigrated with endogenously generated AICD, C60 migrated slightly higher (Fig. 1c). Fe65 cotransfection in the $7 \mathrm{wB} 4$ cells also resulted in stabilization of endogenous AICD (Fig. 1c, compare lanes 1,2). Finally, we confirmed that the AICD generated in 7wB4 cells was a bona fide $\gamma$-secretase product, in that treatment of the cells with a specific and well characterized $\gamma$-secretase inhibitor [2-(di-n-propylamino)tetralin (DAPT) (Dovey et al., 2001)] blocked the formation of this protein (Fig. 1d). Together, these results demonstrate that recombinant C50 and endogenous AICD are closely similar in size and sensitivity to proteases.

A basic prediction of the hypothesis that APP functions as a cell-surface receptor is that the endogenous signaling fragment AICD should be inducible. That is, if AICD mediates a signaling event, its level should exhibit regulation. However, treating our stable APP-transfected $\mathrm{CHO}$ cells $(7 \mathrm{wB} 4)$ with agents known to regulate the $\alpha$-secretase cleavage of the APP ectodomain and thus initiate RIP, e.g., phorbol esters (Buxbaum et al., 1990; Nitsch et al., 1992; Gabuzda et al., 1993; Hung et al., 1993) or crosslinking of the APP ectodomain with antibodies (Rohn et al., 2000; Sudo et al., 2000), did not noticeably alter the level of AICD in these non-neuronal cells (data not shown). We therefore asked whether a role for AICD-mediated signaling might be more readily detectable in neurons, because APP is highly expressed in neurons of organisms from Drosophila to humans (Coulson et al., 2000). Moreover, we wanted to study AICD formation at the endogenous level, because chronic overexpression of APP might interfere with its physiological regulation. Based on this reasoning, we focused our additional experiments on mouse and rat primary neuronal cultures. Plating identical sister cultures from the same batch of pooled embryonic day 18 embryonic brains, we prepared lysates of $10 \mathrm{~cm}$ dishes of these neurons. To minimize the degradation of the highly labile AICD, we lysed the neurons directly in SDS sample buffer and immediately boiled them for 5 min. We then assayed the neuronal cultures over a time period of differentiation spanning 3-11 DIV.

The temporal expression patterns of full-length, endogenous APP (Fig. 2a), its CTFs (Fig. 2b), and AICD (Fig. 2b) in mouse neurons were analyzed by Western blotting with antibody C8. Using this cell model system, several features became apparent. First, levels of full-length APP gradually increased over time (Fig. $2 a$ ), consistent with its known accumulation in neurites, which are under active elaboration in these cultures. Second, AICD consistently accumulated at approximately DIV 6-7 above a baseline amount that was faintly visible at all other time points (DIV 3-5 and 8-11) (Fig. 2b). Third, the fine gel pattern of APP CTFs appeared to shift from lower to slightly higher migrating species (Fig. 2b). This change in APP CTF migration began to occur during the day of peak AICD level (DIV 7) and then persisted as AICD declined (DIV 8-11). We suspected that this change corresponded to phosphorylation, because previous analyses in neurons had described the presence of phosphorylated APP CTFs (Buxbaum et al., 1998a; Lee et al., 2003). We therefore sought to verify that the higher migrating CTF species seen after day 7 were indeed phosphorylated. We subjected lysates at early (DIV 5) and late (DIV 13) time points to dephosphorylation using $\lambda$-PP). Figure $2 c$ shows that a large majority of the CTFs at DIV 5 is not 
phosphorylated, because there is no significant change in the pattern of CTFs after treatment with $\lambda$-PP (Fig. $2 c$, compare lanes 1,2 ) (for a longer exposure, see supplemental Fig. 1, available at www.jneurosci.org as supplemental material). In contrast, at DIV 13, a substantial portion of the CTFs is phosphorylated, because treatment with $\lambda$-PP collapses the five CTF bands to three (Fig. $2 c$, lanes 3,4). The three dephosphorylated bands correspond to the principal recognized CTFs that are present in neurons: $\alpha$-secretase generated C83, and the $\beta$-secretase generated C89 and C99 (Cai et al., 2001). As an additional control, we verified the identity of the putative $\mathrm{C} 83$ and $\mathrm{C} 99$ bands by showing their comigration with transiently overexpressed C83 and C99 (Fig. 2d). Although the five CTFs are difficult to distinguish, the CTF assignments we describe here are in agreement with those reported previously (Buxbaum et al., 1998a).

To analyze more precisely the APP CTFs before and after peak AICD levels, we performed $\lambda$-PP dephosphorylation on four independent batches of primary neurons and analyzed them quantitatively using densitometry. Comparing DIV 5 with DIV 13, we found an approximate twofold increase in total CTFs at the later time point (Fig. $2 e$, left) $(p<0.01)$. This mirrors the gradual rise in full-length APP evident in Figure $2 a$. When we evaluated each CTF individually, we found that, in the DIV 5 neurons, there was $\sim 1.5$ times more C83 and C89 compared with C99 (Fig. 2e, middle). This ratio of individual CTFs was preserved at DIV 13 (Fig. 2e, right), suggesting that the total products of $\alpha$ - and $\beta$-secretase processing are greater at DIV 13 (left) but their relative proportions are unchanged (middle vs right).

We next wanted to confirm the temporal patterns of APP processing and phosphorylation in primary rat neurons, which are significantly easier to culture in robust numbers than are mouse neurons. A very similar pattern of full-length APP, APP CTFs, and AICD was apparent in the rat neuronal cultures (Fig. $3 a, b$ ), demonstrat-

ing that the phenomena described above are shared by neurons from both species. Again, the amount of full-length APP molecules increased progressively over time (Fig. $3 a$ ), whereas AICD levels peaked at approximately DIV 6-7 above a low baseline level (Fig. 3b). Moreover, the APP CTFs altered their migration pattern starting at approximately days 6-7. After this time, we again observed increased levels of slightly higher migrating CTFs. In addition, we noticed that the level of the lowest migrating CTF, C83, rose substantially in association with the increase in AICD at DIV 6 and 7 (Fig. 3b). The same temporal phenomenon was apparent in the mouse neurons (Fig. 2b), suggesting that $\alpha$-secretase cleavage of APP may transiently increase during this

a

b

e
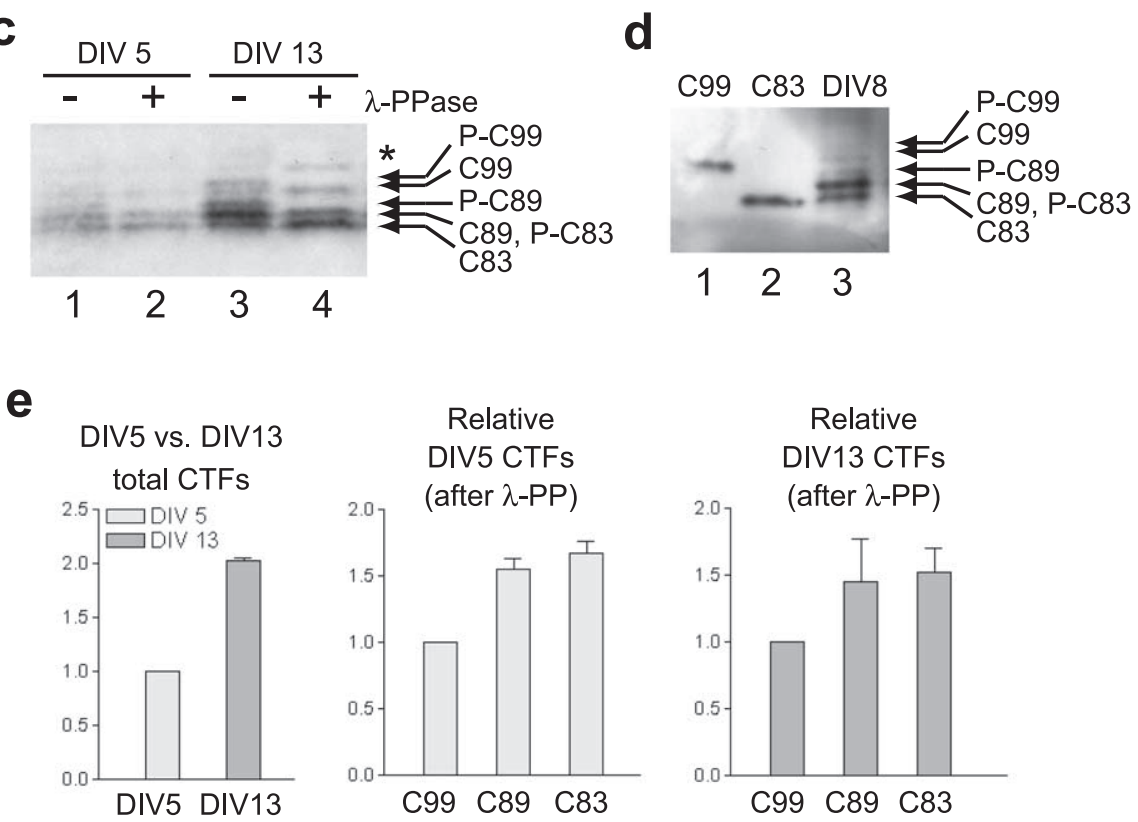

Figure 2. Temporal regulation of APP and its proteolytic fragments in mouse primary neurons. $\boldsymbol{a}$, Expression of full-length APP increases over time in mouse primary neurons. Sister cultures of neurons plated in $10 \mathrm{~cm}$ dishes $\left(1.5 \times 10^{6}\right.$ cells per dish) were collected directly in $2 \times$ Laemmli sample buffer, boiled for $5 \mathrm{~min}$, and sonicated before loading on a $10-20 \%$ Tris-tricine gel for analysis. Nitrocellulose membranes were probed with C 8 antibody. $\boldsymbol{b}$, The same samples as in $\boldsymbol{a}$ were probed for APP CTFs and additional analysis, see Fig. 4). As a migration control, C60 (far left lane) migrated slightly higher than the AICD. The identities of ylation of APP CTFs using $\lambda$-protein phosphatase. Lysates from DIV 5 and DIV 13 neurons were incubated in reaction buffer species. The asterisk represents a nonspecific band as in $\boldsymbol{b}$. $\boldsymbol{d}$, Comigration of the APP CTFs with C83 and C99 identifies these proteins among the five CTF bands. e, Quantitation of relative levels of (83, C89, and C99 at DIV 5 and DIV 13, time points before densitometry after dephosphorylation with $\lambda$-PP. Left, Relative amounts of total CTFs at DIV 5 versus DIV 13. Middle, Within the DIV 5 CTFs, there is $\sim 1.5$ times more $(83$ and $(89$ than C99. Right, This same ratio of C83, C89, and C99 levels is obtained at DIV 13 (although there are twofold more total CTFs compared with DIV 5).

period of neuronal differentiation, resulting in more C83. This result is of particular interest because $\alpha$-secretase has been reported previously to possess both constitutive and inducible activity (Nitsch et al., 1992; Hung et al., 1993; Lammich et al., 1999).

We next examined the subcellular localization of AICD, because we hypothesized that a subset of the fragments should reach nuclei to alter gene transcription. We collected sister cultures of rat primary neurons and subjected them to our previously established nuclear fractionation technique (Kimberly et al., 2001). At all time points, we found that the largest percentage of AICD was localized to the cell membrane fraction (Fig. $3 c$, labeled M). The localization of the APP CTFs to these same M fractions verified 
a

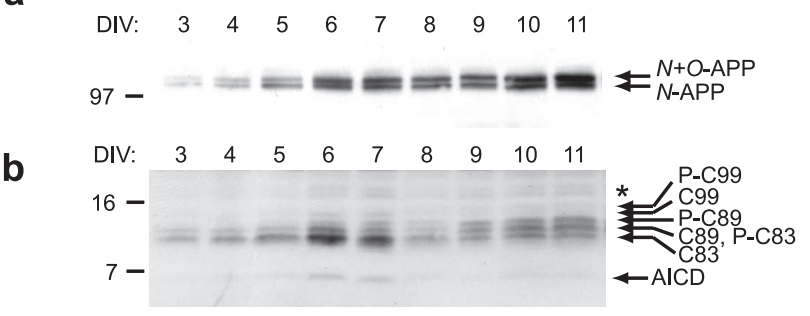

C

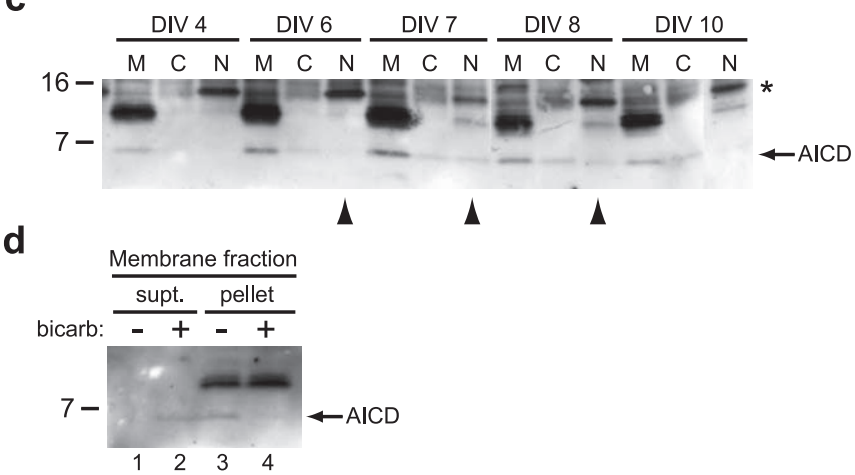

Figure 3. Temporal regulation of APP products and subcellular localization of AICD in rat primary neurons. $\boldsymbol{a}$, A similar analysis in rat primary neurons was performed as in Figure 2 . Identical sister cultures from the same batch were collected at each time point (DIV) and analyzed for full-length APP. With the same number of cells in the culture of each day, the amount of APP protein increased over time. $\boldsymbol{b}$, The APP CTFs and AICD fragments were analyzed, demonstrating a highly similar pattern of transient AICD expression as seen in mouse neurons (Fig. 2b). The transient rise in 83 again correlates with the spike in AICD (DIV 6 and 7). c, Baseline AICD is principally associated with membranes, whereas a portion of the induced AICD translocates into the nuclei. Three $10 \mathrm{~cm}$ plates at each time point were pooled and subjected to fractionation. After addition of detergent and sonication, samples were immunoprecipitated with $\mathrm{C} 8$ and then analyzed by Western blot. M, Membrane; C, cytosol; N, nucleus. The $15 \mathrm{kDa}$ band $\left({ }^{*}\right)$ present in the nuclear fractions is nonspecific, because this band is not confirmed by Western blotting with mouse monoclonal antibody $13 \mathrm{G} 8$ (supplemental Fig. 3, available at www.jneurosci.org as supplemental material). $\boldsymbol{d}$, AICD is loosely associated with membranes. Membrane fractions prepared as in c were washed with buffer or $0.1 \mathrm{~m}$ sodium carbonate, $\mathrm{pH} 11$. The samples were pelleted, and the resultant supernatant (supt.) and pellet were immunoblotted for the presence of AICD. bicarb, Bicarbonate.

the clear enrichment of membranes. Because AICD does not contain a transmembrane domain, its presence in this fraction suggested that it might be associated adventitiously with membranes after its release by $\gamma$-secretase. Consistent with this interpretation, washing the membrane fractions with $0.1 \mathrm{M}$ sodium bicarbonate, pH 11 (Fujiki et al., 1982), resulted in the dissociation of AICD from the membranes into the supernatant (Fig. 3d). In the nuclear fractions $(\mathrm{N})$, we detected a portion of AICD selectively at DIV 6-8 (arrowheads in Fig. 3c). The detection of AICD in nuclei appeared to coincide with the approximate time at which its level in the previous batches of neurons was highest (compare Fig. $3 c$ with Figs. $2 b, 3 b$ ). Slight temporal differences in this pattern (i.e., the presence of nuclear AICD at DIV 8 in Fig. $3 c$ ) reflected the subtle batch-to-batch variability we observed over many experiments, and the ability to detect AICD in the nucleus appeared to vary as a function of its total cellular level. The amount of AICD found in the soluble cytosolic fraction $(\mathrm{C})$ increased at approximately DIV 6 but did not clearly decrease at DIV 10, as did the level of AICD in the nuclei (Fig. 3c). Together, the data in Figure $3 c$ suggest that baseline levels of AICD are associated principally with cellular membranes throughout the time course of neuronal differentiation and that there is a transient peak of AICD generation during which a small portion enters the nucleus. a

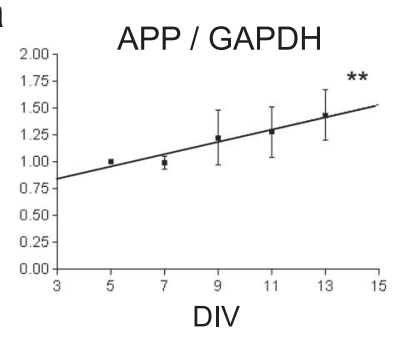

b

C
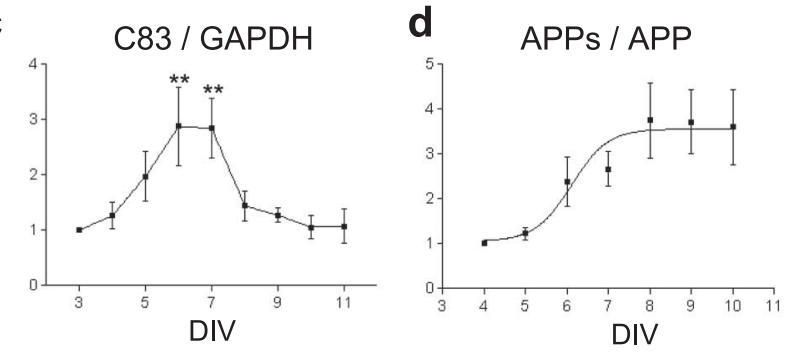

e

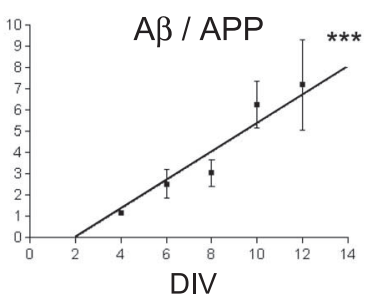

Figure 4. Quantitation of APP and its metabolites in neuronal cultures over time. The levels of various proteins were quantified using Western blotting and film densitometry. For each graph, sister cultures containing the same number of rat neurons were collected at the indicated days after plating. At least three independent batches were quantified for each data point. To normalize for possible batch-to-batch variability, the levels at each time point in a given batch were normalized to the ubiquitous enzyme GAPDH in the same sample. $\boldsymbol{a}$, Full-length APP levels increased over time. ${ }^{* *} p<0.05$ using linear regression analysis. $\boldsymbol{b}$, Quantitation of AICD over time. Note the approximate twofold rise in its level at DIV 6 and 7, which is statistically significant $\left(^{* *} p<0.01\right)$. The increase at DIV 5 was also statistically significant $(p<0.01)$. c, The level of $(83$ exhibits a similar pattern as AICD, with an approximate threefold increase at DIV 6 and 7 $\left.{ }^{* *} p<0.01\right)$. $\boldsymbol{d}, \alpha$-Secretase activity was measured by quantifying APP- $\alpha$ in the conditioned media of four independent batches of neurons. For each data point, media were conditioned for $1 \mathrm{~d}$, and the values were normalized to the relative level of full-length APP. $e, A \beta$ secreted into the medium was quantified using a sensitive and specific sandwich ELISA ( $\left.A \beta_{x-40}\right)$ and normalized to APP levels in the corresponding neuronal lysate. $A \beta$ increased linearly over time $\left.{ }^{* * *} p<0.001\right)$ based on linear regression analysis.

Because the transient elevation and nuclear localization of AICD was a highly consistent finding (observed in $>20$ time course experiments we performed in rat and mouse neurons), we next evaluated what factors might be responsible for this apparent tight temporal regulation. We reasoned that the rise in AICD levels could be attributable, for example, to increased production and/or decreased degradation. Because we observed a rise in C83 just at the time of increased AICD, we carefully quantified the temporal changes by performing densitometric analyses on primary rat neuronal cultures. To compare three different neuronal batches, we plated the same number of neurons in each dish and used media (supplement B27 from Invitrogen) to prevent proliferation of the cells over time. We also normalized each value to the ubiquitous cytoplasmic protein GAPDH to further reduce the effect of dish-to-dish variability. We first quantified full-length APP and found a progressive and statistically significant increase over time in culture (Fig. 4a), in agreement with the qualitative data presented in Figures $2 a$ and $3 a$. We next measured the levels 
of AICD over time and documented a significant twofold rise in its level at DIV 6 and 7 (Fig. 4b). When we assayed C83 (the only unphosphorylated species), we found a closely similar rise at the time corresponding to the AICD peak (Fig. 4c). The subsequent decrease in C83 likely represents the conversion to phosphorylated $\mathrm{C} 83$, which we could not quantify because of its overlap with the $\beta$-secretase product C89 (Fig. $2 c$ ). Therefore, to accurately assess $\alpha$-secretase activity over time, we quantified the amount of secreted APP- $\alpha$ in the conditioned media of the neurons. We conditioned three separate batches of neurons for $24 \mathrm{~h}$ periods between DIV 3 and DIV 10. Because APP gradually increases with time in culture, we normalized each time point to the corresponding amount of APP. Figure $4 d$ demonstrates that there is a rise in $\alpha$-secretase activity at exactly the time when C83 and AICD increase. Moreover, $\alpha$-secretase activity remains high after DIV $6-7$, suggesting that a factor is present in culture that continues to induce its activity. Because there is no evidence to date that $\gamma$-secretase is rate limiting (Esler and Wolfe, 2001), the coordinate increases in C83 and AICD during neuronal differentiation in vitro are likely to be direct consequences of increased $\alpha$-secretase activity.

To explore this explanation further, we directly evaluated the levels and activity of the $\alpha$-, $\beta$-, and $\gamma$-secretases in neurons over time in culture. We quantified the levels of both of the documented $\alpha$-secretases for APP, ADAM10 (Lammich et al., 1999), and TACE (Buxbaum et al., 1998b), as well as the neuronal $\beta$-secretase BACE1 (Vassar et al., 1999). We found no statistically significant changes in the protein levels of any of the three proteases, suggesting that their activity, rather than their abundance, is regulated after DIV 5 (data not shown). We also evaluated the activity of $\gamma$-secretase, because this protease generates AICD from APP CTFs. We focused on $\gamma$-secretase activity per se rather than the levels of presenilin protein, because we had a highly quantitative assay for measuring $\gamma$-activity [amyloid $\beta(\mathrm{A} \beta)$ protein ELISA] (Johnson-Wood et al., 1997) and because endogenous presenilin protein levels are known to be tightly regulated in cells (Thinakaran et al., 1997). We found that the amount of the $\gamma$-secretase product amyloid $\beta$-protein as a function of holoAPP level increased linearly over time (Fig. 4e), indicating that, even at the later days in culture, $\gamma$-secretase capacity was not saturated. This result further indicates that $\gamma$-secretase activity is not downregulated at later time points, removing this explanation for the decrease in steady-state AICD levels consistently observed after days 7-8.

Because the activities of $\alpha$ - and $\gamma$-secretase were high after day 8 but AICD levels were low, we hypothesized that the stability of AICD may be decreased. Because AICD is known to be stabilized by Fe65 (Cao and Sudhof, 2001; Cupers et al., 2001; Kimberly et al., 2001) and degraded by IDE in vitro and in vivo (Edbauer et al., 2002), we tested whether the levels of either of these proteins were altered at later time points. We quantified Fe65 and IDE and found that they were both unchanged over the entire time course of the primary neuronal cultures (data not shown). Therefore, it is unlikely that the level of either of these proteins is mediating the sharp rise and fall of AICD.

To continue the search for factors responsible for suppressing AICD levels at later time points during neuronal differentiation, we next focused on the APP CTF precursors, because we noticed that they became phosphorylated over time (Fig. 2c). Phosphorylation of the C terminus of APP is particularly intriguing because it has been reported to disrupt the interaction with Fe65 (Ando et al., 2001). Because Fe65 markedly stabilizes AICD (Fig. 1), phosphorylation of the APP CTFs could interfere with their interac- tion with Fe65 and diminish the stability of AICD. The C terminus of APP can be phosphorylated at multiple sites, including threonine 654, serine 655, threonine 668 (Oishi et al., 1997), and tyrosine 682 (Russo et al., 2001). Although there is evidence that phosphorylation occurs at each of these sites in various cell lines, we were particularly interested in T668, because this phosphorylation event is neuron specific (Iijima et al., 2000) and because it is the only such modification that has been shown to cause a neuronal phenotype: a defect in neurite extension (Ando et al., 1999). We therefore used a well characterized antibody specific for T668-phosphorylated APP ( $\alpha$ P-T668) (Lee et al., 2003) and probed our neuronal lysates at day 8 , when phosphorylated CTF is present. Figure $5 a$ demonstrates that phosphorylated C83 is recognized by this antibody, whereas unphosphorylated C83 is not (compare lanes 3-5). In the region of the blot in which C89 and $\mathrm{C} 99$ are located, there is an abundant reactive band that obscures their identification (lanes 4,5 ). We therefore evaluated whether this $\alpha \mathrm{P}-\mathrm{T} 668$-reactive band was nonspecific by three methods. First, a careful comparison of size suggests that the band comigrates with the unphosphorylated C99 (Fig. $5 a$, compare lanes 1,5$)$. Second, in three separate $\mathrm{CHO}$ cell lines that do not undergo phosphorylation of APP, $\alpha$ P-T668 antibody nevertheless detects the band (Fig. 5b). It is unlikely that the $\alpha \mathrm{P}-\mathrm{T} 668$ antibody is merely detecting unphosphorylated C99, because the antibody does not cross-react at all with the more abundant C83 and C89 species (Fig. 5b, lanes 5-7). Third, in neuronal lysates that have been precleared of all APP CTFs via C8 immunodepletion (Fig. $5 c$, compare lanes 1,2), $\alpha \mathrm{P}-\mathrm{T} 668$ antibody still detects this band (lane 4). We therefore turned to an IP and Western blotting approach using antibody 13G8 to IP NP-40 lysates from $10 \mathrm{~cm}$ dishes of primary neurons. Figure $5 d$ shows that, at DIV 11 , there is abundant phosphorylation of the APP CTFs (left, lane 2), and detection with the $\alpha \mathrm{P}-\mathrm{T} 668$ antibody reveals two bands consistent with phosphorylated C83 and C89 (right, lane 4). The absence of phosphorylated C99 is likely a reflection of its low abundance. By comparison, there is less phosphorylation at DIV 7 , and the $\alpha \mathrm{P}$-T668 antibody consequently does not appreciably detect any CTFs (Fig. $5 d$, lanes 1,3 ). Therefore, we conclude that endogenous neuronal C83 and C89 are phosphorylated at T668 starting after approximately DIV 7 (Fig. $5 a, d$ ), but we cannot currently determine whether T668 phosphorylation also occurs on C99.

If phosphorylation of T668 in APP CTFs helps abrogate the AICD interaction with Fe65, then the resultant phosphorylated AICD product should be less stable. We therefore examined C50 proteins bearing a Thr to Glu mutation at 668 to mimic the negatively charged phosphate group. Previous studies have used this approach to mimic phosphorylation of APP (Ando et al., 1999) and have specifically shown that either phosphorylation or a negatively charged side chain at this particular site interferes with binding to Fe65 (Ando et al., 2001). As a control, we also made a noncharged Thr to Ala substitution at the same residue. When we transfected COS7 cells with one of these three forms of AICD plus Fe65, we consistently found that the C50 T668E had a substantially lower steady-state level than did either C50 wild type or C50 T668A (Fig. 5e, compare lanes 5, 6 with 1-4). To confirm that this phosphorylation-state mimic results in a destabilization of AICD, we performed coimmunoprecipitations of Fe65 and wild-type or T668E mutant C60. After immunoprecipitation with an antibody to Fe65, the samples were analyzed by Western blotting for Fe 65 and AICD. Figure $5 f$ demonstrates that there is a reduced efficiency in the co-IP of the T668E mutant form of the AICD, demonstrating that this mutation destabilizes 
a

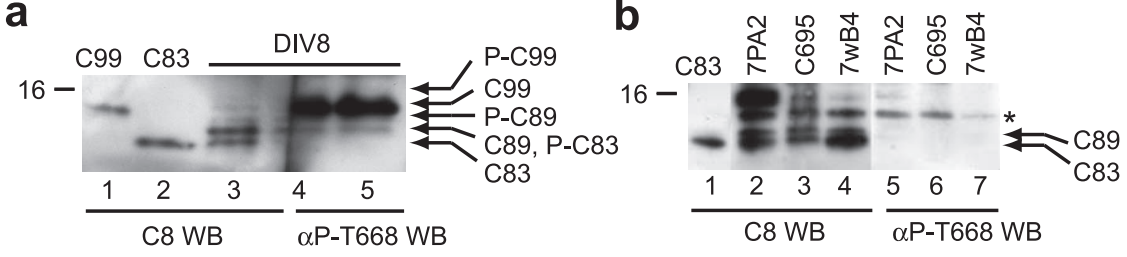

C

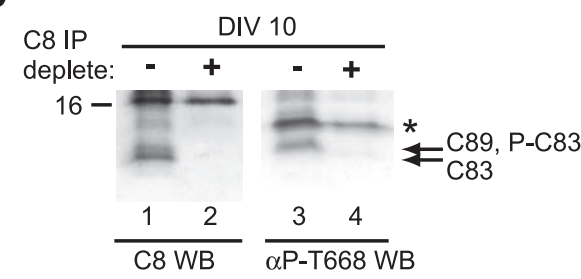

e

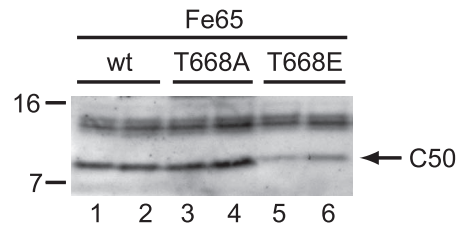

d

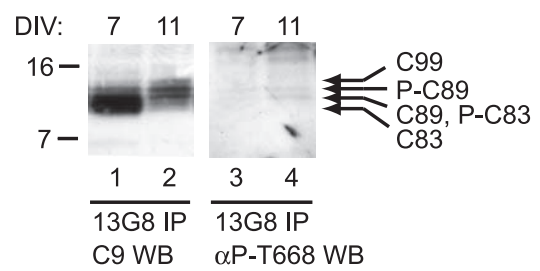

f

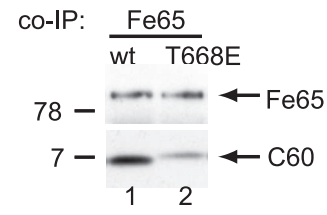

Figure 5. Phosphorylation of APP CTFs at T668 disrupts stabilization by Fe65. $\boldsymbol{a}$, The APP CTF C 83 is phosphorylated at residue Thr668. Rat neuronal lysates were probed with C 8 and the previously characterized $\alpha$ P-T668 antibody (Lee et al., 2003), which is specific for APP phosphorylated at T668. The same sample from a DIV 8 neuronal lysate was run in three adjacent lanes (lanes 3-5). The membrane was cut in the middle of lane 4 , and each half was blotted with the indicated antibody. The dark $\sim 12 \mathrm{kDa}$ band in the $\alpha$ P-T668 Western blot (WB; lanes 4,5) is most consistent with a cross-reactive band (see Results and Fig. $6 b, c) . \boldsymbol{b}$, The $\alpha$ P-T668 antibody detects a cross-reactive band at $\sim 12 \mathrm{kDa}$. The $\alpha \mathrm{P}$-T668 antibody detects a band (asterisk) in the lysates of cells that do not undergo phosphorylation of CTFs: 7PA2, a CHO line stably expressing APP ${ }_{751}$ with V717F mutation; $\mathrm{C}_{695}$, a CHO line stably expressing $\mathrm{APP}_{695}$; and $7 \mathrm{WB} 4$, a $\mathrm{CHO}$ line stably expressing $\mathrm{APP}_{751}$. $\mathbf{c}$, When all CTFs are immunodepleted from a rat neuronal lysate by $(8$, the nonspecific band remains present in the post-IP supernatant (lanes 3, 4, marked by an asterisk). In the left panel, the supernatants are detected with $(8$, demonstrating that the APP CTFs are present in the mock immunodepleted sample $(-)$ but not in the (8-depleted sample $(+)$. In the right panel, $\alpha \mathrm{P}$-T668 antibody detects the cross-reactive band in both samples. $\boldsymbol{d}$, Immunoprecipitation and Western blotting reveals phosphorylated C 83 and $(89$ at T668. Neuronal lysates were subjected to IP with 13G8 and then analyzed by Western blotting with either (9 (left) or $\alpha$ P-T668 antibody (right). Using this technique, the cross-reactive band is absent, but the abundance of $(99$ is too low to evaluate. $\boldsymbol{e}$, A negative charge at Thr668 disrupts the stabilization of ( 50 by Fe65. Whereas the levels of wild-type (wt) and Thr to Ala mutant (T668A) C50 are essentially identical, the amount of (50 Thr to Glu (T668E) is substantially diminished (duplicate samples run side-by-side). $\boldsymbol{f}$, A negative charge at Thr668 reduces the interaction between Fe65 and C60. COS cells were transfected with Fe65 and either wild-type (lanes 1,2) or mutant (lanes 3, 4) T668E C60. After lysis with NP-40 lysis buffer, Fe65 was immunoprecipitated, and the resulting IPs were Western blotted for Fe65 and APP. Duplicate samples are shown.

the interaction with Fe65 resulting in diminished steady-state levels. There was an excess of C60 protein in the supernatants of both wild-type and $\mathrm{T} \rightarrow \mathrm{E}$ (threonine $\rightarrow$ glutamate) samples (supplemental Fig. 2, available at www.jneurosci.org as supplemental material), suggesting that the difference in the coimmunoprecipitation represents a difference in the binding to Fe65. Together, these data demonstrate that mutation at T668 to mimic phosphorylation results in lower steady-state levels of the protein, a result entirely consistent with previous work (Ando et al., 2001).

We next sought to identify the protein kinase that is responsible for phosphorylating APP at T668 in neurons, because its identity may shed light on the physiological regulation and function of APP. Several candidate kinases have been reported to phosphorylate APP, including two neuron-specific kinases: cyclin-dependent kinase 5 (cdk5) and JNK3 (also called SAPK $\beta$ p54) (Standen et al., 2001). Both kinases are of interest in that they have been reported to have roles in mediating neuronal death (Yang et al., 1997; Patrick et al., 1999) and also normal neuronal function (Chae et al., 1997; Coffey et al., 2000; Waetzig and Herdegen, 2003). Transfecting cdk5 with either its p25 or p35 activator into multiple cell lines only modestly phosphorylated full-length APP (data not shown), consistent with previous observations that this kinase can phosphorylate APP (Iijima et al., 2000). However, we could not detect any phosphorylated CTFs as we had observed in our primary neurons. Therefore, we explored other kinases that might be responsible for phosphorylation in the primary neurons. When we transfected JNK3 into a cell line that has minimal phosphorylation of APP CTFs (COS7 cells), we observed a pattern of APP CTF bands that mimicked that of the primary neurons after DIV 7 (Fig. 6a). We next confirmed that this change in migration was attributable to phosphorylation at T668, using the $\alpha \mathrm{P}-\mathrm{T} 668$ antibody (Fig. 6b). As it did in the primary neurons, this antibody specifically detected phosphorylated C83 (Fig. 6b, lanes 2, 4). Because of the low abundance of C89 and C99, we could make no conclusions about its phosphorylation state. The finding that JNK3, but not cdk5, was able to avidly phosphorylate APP in transfected cells is consistent with in vitro studies (Standen et al., 2001). Importantly, we observed a mechanistic connection between JNK3 phosphorylation and APP signaling function, in that we noticed a decrease in the steady-state levels of AICD during JNK3 coexpression (Fig. $6 c$, lanes 2, 4). This is valid because these COS cells express Fe65 endogenously (supplemental Fig. 4, available at www.jneurosci.org as supplemental material). This finding indicates that T668 phosphorylation by JNK3 reduces AICD levels in cells.

Because we were able to induce the phosphorylation of APP at T668 in nonneural cells and decrease AICD levels as a result, we returned to the rat primary neurons to search for any temporal change in endogenous JNK3 activity. In brain, JNK3 exists in two alternatively spliced isoforms (with and without a C-terminal extension) that generate a 54 and a $46 \mathrm{kDa}$ protein, respectively (Davis, 2000). Although the significance of the isoforms is unknown, we probed for the levels of these two proteins in our rat primary neurons over the time course of differentiation. We found an increase in the levels of both JNK3 isoforms at approximately DIV 7 (Fig. 6d, top). JNK3 kinase activity is itself regulated by phosphorylation of its Thr-Pro-Tyr motif (Davis, 2000). We therefore used an antibody specific for all activated forms of JNK and found that the phospho-JNK-p54 and -p46 proteins increased sharply at approximately DIV 7 (Fig. $6 d$, middle). This event was followed immediately by the onset of APP phosphorylation at T668, the upshift in the migration of $\mathrm{C} 83$, and the decline in AICD (Fig. 6d, bottom). Therefore, not only can T668 phosphorylation be induced in non-neural cells by JNK3 overexpression, but the endogenous levels and activity of this kinase 
correlate with T668 phosphorylation of endogenous APP in primary neurons.

JNK3 is one of three genes in the JNK family. Previous reports had shown that JNK1 could phosphorylate full-length APP in vitro and in cell culture. Moreover, the JNK kinases can be activated by stress. Therefore, to evaluate APP phosphorylation under these conditions, we compared the transient expression of JNK1, JNK2, and JNK3 with and without the induction of cellular stress via treatment with sorbitol for $30 \mathrm{~min}$. In each case, we had robust expression of full-length APP and APP CTFs (Fig. 7a, left). Moreover, we found the presence of a higher migrating CTF in the presence of JNK3 and sorbitol (Fig. 7a, left, lane 8). We next probed the same samples with the $\alpha \mathrm{P}-\mathrm{T} 668$ antibody (Fig. 7a, right). Consistent with previous reports, we demonstrated that JNK1 was able to phosphorylate full-length APP in the presence of cellular stress (Fig. 7a, right, lanes 3, 4). This resulted in a modest increase in APP CTF phosphorylation. JNK2 was also able to phosphorylate full-length APP and, to an even less efficient degree, APP CTFs (Fig. 7a, lanes 5, 6). However, JNK3 expression robustly phosphorylated full-length APP and APP CTFs to much more so than either JNK1 or JNK2. The fact that JNK3 could phosphorylate APP in the absence of sorbitol (Figs. $6 a-c, 7 a$ ) is likely a reflection of cellular stress in the presence of the liposomal transfection reagent (which is known to cause cell death). Because JNK1 and JNK2 were able to phosphorylate APP, albeit less efficiently than JNK3, we evaluated the effect on AICD levels. Figure $7 b$ shows that, despite adequate expression of JNK1 and JNK2, there is no effect on AICD levels. Together, these data demonstrate that JNK3 is the most effective kinase to phosphorylate APP at T668 compared with cdk5, JNK1, and JNK2. It is also the only kinase that can sufficiently phosphorylate APP and APP CTFs to lower AICD levels.

Because these experiments are the result of transient overexpression, we sought to confirm the role of JNK3 in vivo. We therefore evaluated the phosphorylation of APP and the level of AICD in mouse brain lysates from wild-type and JNK3-ablated animals (Yang et al., 1997). When we probed for the APP CTFs, we observed a reduction in the amount of phosphorylated CTFs in the JNK3 ${ }^{-/-}$lysate when compared with identically prepared wild-type brain lysates (Fig. 7c). We compared that with the effect of ablation of JNK1 and JNK2. Consistent with our results in protein overexpression, the absence of JNK1 or JNK2 in vivo did not appreciably change the pattern of APP CTFs (Fig. $7 d$ ). We then examined longer exposures for the presence of AICD and found an increase in its level only in the $\mathrm{JNK} 3^{-1-}$ brain lysate (Fig. 7e, compare lanes 7, 8 with 1-6). Because the effect was not dramatic, we quantified the levels from six pairs of brains from seven separate experiments and found a statistically significant 1.51 -fold increase $(p<0.005)$ in AICD in the JNK3 ${ }^{-1-}$ lysate compared with wild-type brain. We further evaluated the level of b

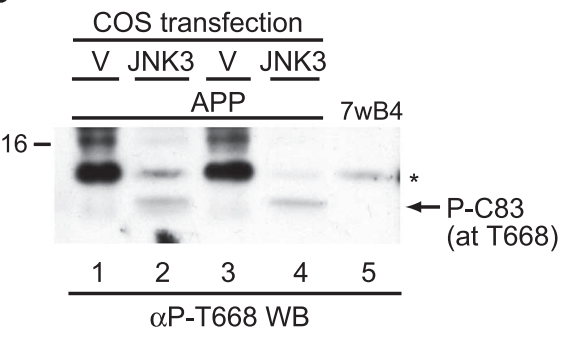

d

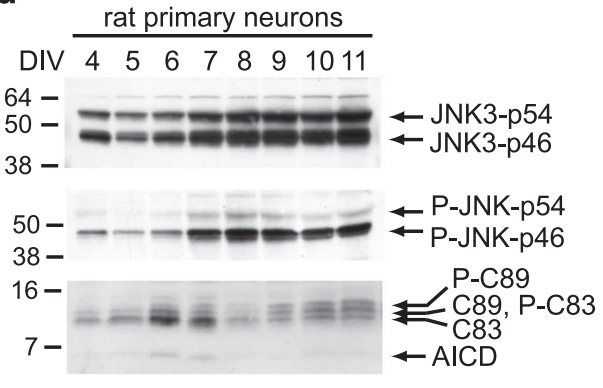

Figure 6. JNK3 phosphorylates APP at T668 and destabilizes AICD. $\boldsymbol{a}$, JNK3 transfection results in phosphorylation of APP CTFs. in the amount of AICD, which was now undetectable. $\boldsymbol{d}$, JNK3 levels and phosphorylation increase during the primary neuron time course. Rat neuronal lysate was probed with a JNK3-specific antibody that detects both splice isoforms of this protein (top). The amount of active (phosphorylated) JNK was detected using a phosphorylation statespecific antibody (middle). The time course for APP (TFs and AICD is shown for visual reference (bottom).

AICD in primary neurons cultured from $\mathrm{JNK} 3^{-1-}$ brain. We found that the steady-state level of AICD in JNK3 ${ }^{-1-}$ DIV 4 neurons was higher than that of equivalent lysates from wild-type neurons (Fig. $7 f$ ), supporting the results in the whole-brain lysates. Interestingly, the JNK3 ${ }^{-1-}$ neurons consistently had lower levels of full-length APP (Fig. $7 f$, top). This may suggest that nonphosphorylated APP may be a better substrate for $\alpha$ - and/or $\beta$-secretase. Nevertheless, the level of AICD is higher in the JNK $3^{-1-}$ compared with wild type, despite the decreased amount of full-length APP. Finally, we evaluated APP CTFs and AICD levels in wild-type and $\mathrm{cdk} 5^{-1-}$ brain lysate and found no detectable differences (data not shown). Together, these data confirm in vivo that JNK3 in the major kinase for APP at T668 and that this activity regulates the level of AICD. Our results strongly suggest that JNK3 is responsible for decreasing the level of AICD during neuronal differentiation, raising the possibility that it regulates APP signaling.

\section{Discussion}

Based on analogy to Notch and other substrates of $\gamma$-secretase, APP has been hypothesized to function as a cell-surface receptor that signals via the proteolytic release of the AICD fragment (Ebinu and Yankner, 2002). We now provide evidence for this hypothesis by detecting and analyzing endogenous AICD in primary neurons. We find that AICD is temporally regulated, and its 
a

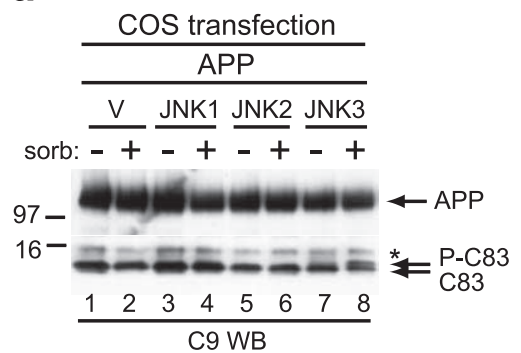

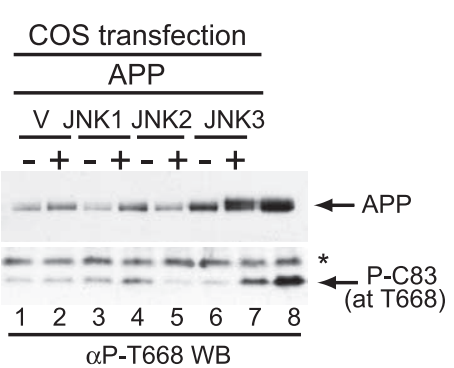

b

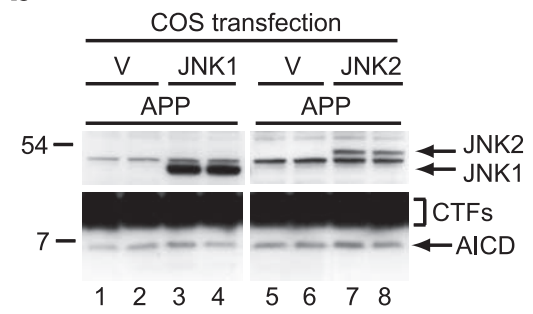

C

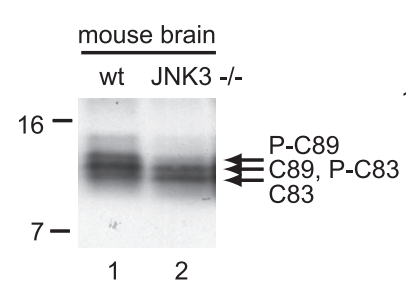

d

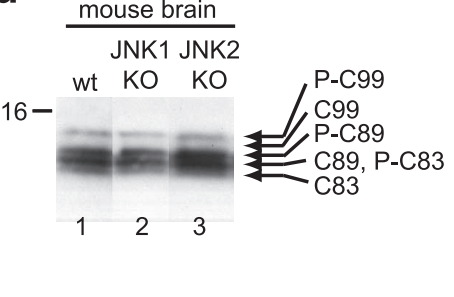

e

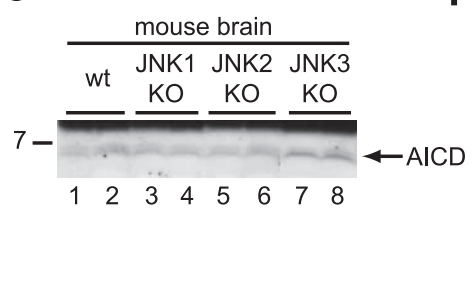

f

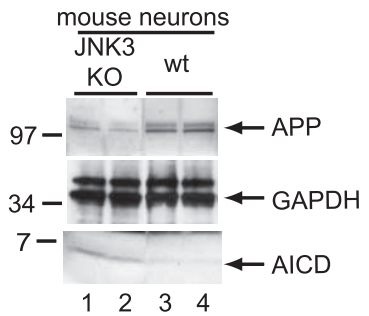

Figure 7. JNK3 is the principal kinase for APP in vitro and in vivo. $\boldsymbol{a}$, JNK3 phosphorylates APP more avidly than JNK1 or JNK2. COS7 cells were transfected with APP 695 and the indicated JNK constructs. The cells were then treated with $10 \mu \mathrm{m}$ sorbitol (sorb) or vehicle control 30 min before collection. Lysates were probed with a C-terminal-specific anti-APP antibody (C9, left) or a T668 phosphorylation-specific anti-APP antibody (right). $\boldsymbol{b}$, JNK1 or JNK2 overexpression does not affect the level of AICD. COS7 cells were transfected with APP and either vector JNK1 or JNK2 plasmids, and the resulting lysate was probed for JNK expression (top), APP (middle), and AICD (bottom). V, Vector. c, JNK3 is required for APP phosphorylation in vivo in mouse brain. Protein (30 $\mu$ g) was loaded in the B6 wild-type (wt) and JNK3 ${ }^{-1-}$ lanes and then detected with polyclonal ( 9 antibody. $d$, The absence of JNK1 or JNK2 does not have any effect on APP (TFs. Protein ( $30 \mu \mathrm{g}$ ) was loaded into each lane of a $10-20 \%$ Tris-tricine gel and then probed for APP CTFs. e, Only JNK3 has an effect on AICD levels in mouse brain. Mouse brain lysates were prepared as in c for wild-type, JNK1 - - JNK2 ${ }^{-1-}$, and JNK3 ${ }^{-1-}$ brains. Antibody $\left(9\right.$ was used for detection of AICD. $f$, Steady-state levels of AICD are elevated in JNK3 ${ }^{-1-}$ mouse neurons compared with wild-type control. DIV 4 neurons were prepared and analyzed as in Figure $2, a$ and $b$. The amount of full-length APP was slightly lower in the JNK3 ${ }^{-/-}$neurons; nevertheless, the level of AICD was elevated. This is consistent with the results in total mouse brain lysates. KO, Knock-out; WB, Western blot.

rise and fall correlates with the levels of its precursor, the APP CTF C83. Furthermore, we show that a portion of AICD localizes to the nucleus during this time, positioning it for a role in altering gene transcription. Although evaluation of AICD in overexpressed heterologous reporter systems suggest that AICD may be dispensable in the nucleus for gene transactivation (Cao and Sudhof, 2004), our data suggest that a portion of AICD may nevertheless translocate there. After DIV 7, we also show that JNK3mediated phosphorylation of the APP CTFs (specifically at T668) disrupts the stabilization of AICD by Fe65 and suppresses AICD levels. Together, our data identify specific mechanisms for the tight regulation of APP signaling under endogenous conditions during neuronal differentiation.

\section{AICD as a regulated signaling molecule}

Although the analogy to Notch and other $\gamma$-secretase substrates has provided insight into the putative function of APP, several aspects of APP biology have stood in apparent contradiction. For a signaling receptor, a basic feature is the ability to promote or discontinue signaling through an interaction with its ligand. For Notch, its ligand and the regulated formation of Notch intracellular domain have been well described (Mumm and Kopan, 2000). In contrast, the APP ligand is still unknown, and the detection of endogenous AICD and any regulation of its level have proved challenging. Our description of endogenous neuronal AICD and its regulation in primary neurons now resolves those contradictions. Although AICD function and biology has been challenging to study, our data raise the possibility that one of the functions of APP may be mediated through the release of AICD.

Our analyses in both mouse and rat primary neurons also provide indirect evidence for the existence of a ligand. Signaling through the $\gamma$-secretase hub occurs through stepwise proteolysis of various receptors, beginning with a ligand-activated initial cleavage mediated by an ADAM protease (Kimberly and Wolfe, 2003). For APP, that ADAM (or $\alpha$-secretase) cleavage results in the formation of C83. Although we have no direct evidence for the identity of an APP ligand, we do observe a twofold rise in C83, which we postulate is a result of an APP-ligand interaction starting at approximately day 6 during neuronal differentiation in vitro. A ligand activation of $\alpha$-secretase that yields C83 (compared with $\beta$-secretase yielding C99) is particularly appealing, because a variety of reports have demonstrated that $\alpha$-secretase is inducible (Buxbaum et al., 1990; Nitsch et al., 1992; Hung et al., 1993; Lammich et al., 1999). Finally, our description of a specific time during which $\alpha$-secretase is naturally modulated in neurons provides a cell model system in which to begin the screening and identification of the cognate APP ligand.

\section{Phosphorylation by JNK3 at T668 suppresses AICD levels after approximately day 7 in vitro}

Induction of $\alpha$-secretase activity (via ligand interaction) is not the only potential mechanism by which AICD signaling is regulated. At later time points in culture, the total amount of CTFs continues to increase (Fig. $2 e$, left), and yet the total amount of AICD returns to its low baseline (Fig. $4 b$ ). This phenomenon is not attributable to a limitation of $\gamma$-secretase activity, because robust amounts of the other $\alpha$ and $\gamma$ products (APP- $\alpha$ and A $\beta$, respectively) remain high (Fig. $4 d, e$ ). Therefore, some other mechanism suppresses the amount of steady-state AICD present in the neurons. We show that the phosphorylation of the APP CTFs is well correlated with the temporal decrease in AICD. In particular, we find that phosphorylation occurs at T668, which is part of the TPEE motif in the APP cytoplasmic domain. This motif has been found to contain transient structure by solution nuclear magnetic resonance spectroscopy (Kroenke et al., 1997; Ramelot et al., 2000). Importantly, phosphorylation of T668 sig- 
nificantly alters that conformation, suggesting that phosphorylation may act as a conformational switch in the binding of cytoplasmic partners by APP (Ramelot and Nicholson, 2001). Indeed, Fe65 binds to the APP cytoplasmic domain between the TPEE and the NPTY motifs, and phosphorylation at T668 diminishes the efficiency of that interaction (Ando et al., 2001). In accord, we use mutagenesis at T668 to confirm that addition of a negative charge results in the marked destabilization of AICD (Fig. 5e,f). Thus, when Fe65 can interact with APP, it protects AICD from degradation. However, phosphorylated APP cannot interact with Fe65 as avidly, and AICD levels accordingly decrease.

We also identify here the kinase that is principally responsible for this suppression of AICD levels after DIV 7. JNK3 is a neuronspecific member of the JNK family of mitogen-activated protein kinases. Whereas it was initially shown to play a role in the neuron stress response (Yang et al., 1997), more recently JNK3 has been demonstrated to play important roles in neuronal differentiation (Coffey et al., 2000; Waetzig and Herdegen, 2003). Our discovery that JNK3 regulates AICD levels at a key point in neuronal differentiation further supports this notion. Although it remains possible that cdk5, JNK1, JNK2, or other kinases may also play a role in APP phosphorylation and AICD regulation, the data presented herein suggest that JNK3 plays a major role.

Together with previous studies, our data therefore provide a specific model for the physiological regulation of AICD signaling in neurons. An unidentified ligand activates $\alpha$-secretase activity at approximately day 5 or 6 during neuronal differentiation. This induces an increase in the production of C83 and AICD, and a portion of the latter translocates to the nucleus, in which it may affect nuclear transcription in conjunction with Fe65. However, because total APP CTFs continue to gradually increase over time in culture (Fig. 2e, left), a mechanism for suppression of AICD is apparently needed. Activation of the JNK3 cascade results in phosphorylation of APP at T668 starting at approximately DIV 7 or 8. This phosphate destabilizes the interaction with Fe65 and thus suppresses the level of AICD, despite an additional rise in the CTF precursors.

\section{Implications for the function of AICD in primary neurons}

The discovery of a precise regulation of endogenous AICD during neuronal differentiation provides evidence of a signaling function of APP. In $>20$ different batches of primary neurons we examined, we consistently saw a quantifiable accumulation of AICD at DIV 5-7. This result suggests that a maturational event occurring at approximately 1 week in culture is regulated, at least in part, by AICD signaling. Comparative analyses among several model organisms provide circumstantial evidence that APP (and perhaps AICD, in particular) plays a role in neurite outgrowth, in the genesis and/or remodeling of synapses, and in neuronal organization. Spatial and temporal analyses of neurons support an axonal and synaptic distribution of APP. APP has been directly visualized in a subset of synapses within human and rat brain, at the neuromuscular junction (Schubert et al., 1991), and in the fly, in specific layers of the optic lobes in which synapses are actively forming (Torroja et al., 1996). Increases in APP secretion appear to occur at the time of end-arbor formation in rat brain (Moya et al., 1994). More recently, ablation of all three APP family members in the mouse resulted in neuronal ectopia and altered synaptophysin staining (Herms et al., 2004).

Approaches by our laboratory to identify molecular targets through transcriptional profiling have yet to yield a unified function of AICD (D. Walsh and D. Selkoe, unpublished observations). Alternative approaches, such as manipulating AICD levels in neurons and assessing the effect on synapses and neurite outgrowth, will require significant time but may eventually prove fruitful. Nevertheless, our current data provide substantial strides in understanding the physiological regulation of AICD by JNK3 during neuronal differentiation and suggest that these proteins mediate an aspect of synaptogenesis.

\section{References}

Ando K, Oishi M, Takeda S, Iijima K, Isohara T, Nairn AC, Kirino Y, Greengard P, Suzuki T (1999) Role of phosphorylation of Alzheimer's amyloid precursor protein during neuronal differentiation. J Neurosci 19:4421-4427.

Ando K, Iijima KI, Elliott JI, Kirino Y, Suzuki T (2001) Phosphorylationdependent regulation of the interaction of amyloid precursor protein with Fe65 affects the production of beta-amyloid. J Biol Chem 276:40353-40361.

Artavanis-Tsakonas S, Rand MD, Lake RJ (1999) Notch signaling: cell fate control and signal integration in development. Science 284:770-776.

Brown MS, Ye J, Rawson RB, Goldstein JL (2000) Regulated intramembrane proteolysis: a control mechanism conserved from bacteria to humans. Cell 100:391-398.

Buxbaum JD, Gandy SE, Cicchetti P, Ehrlich ME, Czernik AJ, Fracasso RP, Ramabhadran TV, Unterbeck AJ, Greengard P (1990) Processing of Alzheimer $\beta / A 4$ amyloid precursor protein: Modulation by agents that regulate protein phosphorylation. Proc Natl Acad Sci USA 87:6003-6006.

Buxbaum JD, Thinakaran G, Koliatsos V, O'Callahan J, Slunt HH, Price DL, Sisodia SS (1998a) Alzheimer amyloid protein precursor in the rat hippocampus: transport and processing through the perforant path. J Neurosci 18:9629-9637.

Buxbaum JD, Liu KN, Luo Y, Slack JL, Stocking KL, Peschon JJ, Johnson RS, Castner BJ, Cerretti DP, Black RA (1998b) Evidence that tumor necrosis factor alpha converting enzyme is involved in regulated alpha-secretase cleavage of the Alzheimer amyloid protein precursor. J Biol Chem 273:27765-27767.

Cai H, Wang Y, McCarthy D, Wen H, Borchelt DR, Price DL, Wong PC (2001) BACE1 is the major beta-secretase for generation of Abeta peptides by neurons. Nat Neurosci 4:233-234.

Cao X, Sudhof TC (2001) A transcriptionally active complex of APP with Fe65 and histone acetyltransferase Tip60. Science 293:115-120.

Cao X, Sudhof TC (2004) Dissection of amyloid-beta precursor proteindependent transcriptional transactivation. J Biol Chem 279:24601-24611.

Chae T, Kwon YT, Bronson R, Dikkes P, Li E, Tsai LH (1997) Mice lacking p35, a neuronal specific activator of Cdk5, display cortical lamination defects, seizures, and adult lethality. Neuron 18:29-42.

Coffey ET, Hongisto V, Dickens M, Davis RJ, Courtney MJ (2000) Dual roles for c-Jun N-terminal kinase in developmental and stress responses in cerebellar granule neurons. J Neurosci 20:7602-7613.

Coulson EJ, Paliga K, Beyreuther K, Masters CL (2000) What the evolution of the amyloid protein precursor supergene family tells us about its function. Neurochem Int 36:175-184.

Cupers P, Orlans I, Craessaerts K, Annaert W, De Strooper B (2001) The amyloid precursor protein (APP)-cytoplasmic fragment generated by gamma-secretase is rapidly degraded but distributes partially in a nuclear fraction of neurones in culture. J Neurochem 78:1168-1178.

Davis RJ (2000) Signal transduction by the JNK group of MAP kinases. Cell 103:239-252.

Dawson GR, Seabrook GR, Zheng H, Smith DW, Graham S, O’Dowd G, Bowery BJ, Boyce S, Trumbauer ME, Chen HY, Van der Ploeg LH, Sirinathsinghji DJ (1999) Age-related cognitive deficits, impaired longterm potentiation and reduction in synaptic marker density in mice lacking the beta-amyloid precursor protein. Neuroscience 90:1-13.

Dovey HF, John V, Anderson JP, Chen LZ, de Saint Andrieu P, Fang LY, Freedman SB, Folmer B, Goldbach E, Holsztynska EJ, Hu KL, JohnsonWood KL, Kennedy SL, Kholodenko D, Knops JE, Latimer LH, Lee M, Liao Z, Lieberburg IM, Motter RN, et al. (2001) Functional gammasecretase inhibitors reduce beta-amyloid peptide levels in brain. J Neurochem 76:173-181.

Ebinu JO, Yankner BA (2002) A RIP tide in neuronal signal transduction. Neuron 34:499-502.

Edbauer D, Willem M, Lammich S, Steiner H, Haass C (2002) Insulindegrading enzyme rapidly removes the beta-amyloid precursor protein intracellular domain (AICD). J Biol Chem 277:13389-13393. 
Esler WP, Wolfe MS (2001) A portrait of Alzheimer secretases-new features and familiar faces. Science 293:1449-1454.

Farris W, Mansourian S, Chang Y, Lindsley L, Eckman EA, Frosch MP, Eckman CB, Tanzi RE, Selkoe DJ, Guenette S (2003) Insulin-degrading enzyme regulates the levels of insulin, amyloid beta-protein, and the betaamyloid precursor protein intracellular domain in vivo. Proc Natl Acad Sci USA 100:4162-4167.

Fujiki Y, Hubbard AL, Fowler S, Lazarow PB (1982) Isolation of intracellular membranes by means of sodium carbonate treatment: application to endoplasmic reticulum. J Cell Biol 93:97-102.

Gabuzda D, Busciglio J, Yankner BA (1993) Inhibition of beta-amyloid production by activation of protein kinase C. J Neurochem 61:2326-2329.

Gao Y, Pimplikar SW (2001) The gamma-secretase-cleaved C-terminal fragment of amyloid precursor protein mediates signaling to the nucleus. Proc Natl Acad Sci USA 98:14979-14984.

Gu Y, Misonou H, Sato T, Dohmae N, Takio K, Ihara Y (2001) Distinct intramembrane cleavage of the beta-amyloid precursor protein family resembling gamma-secretase-like cleavage of Notch. J Biol Chem 276:35235-35238.

Haass C, Schlossmacher MG, Hung AY, Vigo-Pelfrey C, Mellon A, Ostaszewski BL, Lieberburg I, Koo EH, Schenk D, Teplow DB, Selkoe DJ (1992) Amyloid $\beta$-peptide is produced by cultured cells during normal metabolism. Nature 359:322-325.

Herms J, Anliker B, Heber S, Ring S, Fuhrmann M, Kretzschmar H, Sisodia S, Muller U (2004) Cortical dysplasia resembling human type 2 lissencephaly in mice lacking all three APP family members. EMBO J 23:4106-4115.

Hung AY, Haass C, Nitsch RM, Qiu WQ, Citron M, Wurtman RJ, Growdon JH, Selkoe DJ (1993) Activation of protein kinase C inhibits cellular production of the amyloid b-protein. J Biol Chem 268:22959-22962.

Iijima K, Ando K, Takeda S, Satoh Y, Seki T, Itohara S, Greengard P, Kirino Y, Nairn AC, Suzuki T (2000) Neuron-specific phosphorylation of Alzheimer's beta-amyloid precursor protein by cyclin-dependent kinase 5 . J Neurochem 75:1085-1091.

Johnson-Wood K, Lee M, Motter R, Hu K, Gordon G, Barbour R, Khan K, Gordon M, Tan H, Games D, Lieberburg I, Schenk D, Seubert P, McConlogue L (1997) Amyloid precursor protein processing and A beta42 deposition in a transgenic mouse model of Alzheimer disease. Proc Natl Acad Sci USA 94:1550-1555.

Kimberly WT, Wolfe MS (2003) Identity and function of gamma-secretase. J Neurosci Res 74:353-360.

Kimberly WT, Zheng JB, Guenette SY, Selkoe DJ (2001) The intracellular domain of the beta-amyloid precursor protein is stabilized by Fe65 and translocates to the nucleus in a notch-like manner. J Biol Chem 276:40288-40292.

Koo EH, Squazzo SL (1994) Evidence that production and release of amyloid beta-protein involves the endocytic pathway. J Biol Chem 269:17386-17389.

Kroenke CD, Ziemnicka-Kotula D, Xu J, Kotula L, Palmer III AG (1997) Solution conformations of a peptide containing the cytoplasmic domain sequence of the beta amyloid precursor protein. Biochemistry 36:8145-8152.

Lammich S, Kojro E, Postina R, Gilbert S, Pfeiffer R, Jasionowski M, Haass C, Fahrenholz F (1999) Constitutive and regulated alpha-secretase cleavage of Alzheimer's amyloid precursor protein by a disintegrin metalloprotease. Proc Natl Acad Sci USA 96:3922-3927.

Lee MS, Kao SC, Lemere CA, Xia W, Tseng HC, Zhou Y, Neve R, Ahlijanian MK, Tsai LH (2003) APP processing is regulated by cytoplasmic phosphorylation. J Cell Biol 163:83-95.

Moya KL, Benowitz LI, Schneider GE, Allinquant B (1994) The amyloid precursor protein is developmentally regulated and correlated with synaptogenesis. Dev Biol 161:597-603.

Mucke L, Masliah E, Johnson WB, Ruppe MD, Alford M, Rockenstein EM, Forss-Petter S, Pietropaolo M, Mallory M, Abraham CR (1994) Synaptotrophic effects of human amyloid beta protein precursors in the cortex of transgenic mice. Brain Res 666:151-167.

Mumm JS, Kopan R (2000) Notch signaling: from the outside in. Dev Biol 228:151-165.

Nitsch RM, Slack BE, Wurtman RJ, Growdon JH (1992) Release of Alzheimer amyloid precursor derivatives stimulated by activation of muscarinic acetylcholine receptors. Science 258:304-307.

Oishi M, Nairn AC, Czernik AJ, Lim GS, Isohara T, Gandy SE, Greengard P, Suzuki T (1997) The cytoplasmic domain of Alzheimer's amyloid pre- cursor protein is phosphorylated at Thr654, Ser655, and Thr668 in adult rat brain and cultured cells. Mol Med 3:111-123.

Patrick GN, Zukerberg L, Nikolic M, de la Monte S, Dikkes P, Tsai LH (1999) Conversion of $\mathrm{p} 35$ to $\mathrm{p} 25$ deregulates Cdk5 activity and promotes neurodegeneration. Nature 402:615-622.

Podlisny MB, Ostaszewski BL, Squazzo SL, Koo EH, Rydell RE, Teplow DB, Selkoe DJ (1995) Aggregation of secreted amyloid beta-protein into sodium dodecyl sulfate-stable oligomers in cell culture. J Biol Chem 270:9564-9570.

Ramelot TA, Nicholson LK (2001) Phosphorylation-induced structural changes in the amyloid precursor protein cytoplasmic tail detected by NMR. J Mol Biol 307:871-884.

Ramelot TA, Gentile LN, Nicholson LK (2000) Transient structure of the amyloid precursor protein cytoplasmic tail indicates preordering of structure for binding to cytosolic factors. Biochemistry 39:2714-2725.

Rohn TT, Ivins KJ, Bahr BA, Cotman CW, Cribbs DH (2000) A monoclonal antibody to amyloid precursor protein induces neuronal apoptosis. J Neurochem 74:2331-2342.

Roncarati R, Sestan N, Scheinfeld MH, Berechid BE, Lopez PA, Meucci O, McGlade JC, Rakic P, D'Adamio L (2002) The gamma-secretase-generated intracellular domain of beta-amyloid precursor protein binds Numb and inhibits Notch signaling. Proc Natl Acad Sci USA 99:7102-7107.

Russo C, Salis S, Dolcini V, Venezia V, Song XH, Teller JK, Schettini G (2001) Amino-terminal modification and tyrosine phosphorylation of [corrected] carboxy-terminal fragments of the amyloid precursor protein in Alzheimer's disease and Down's syndrome brain. Neurobiol Dis 8:173-180.

Sastre M, Steiner H, Fuchs K, Capell A, Multhaup G, Condron MM, Teplow DB, Haass C (2001) Presenilin-dependent gamma-secretase processing of beta-amyloid precursor protein at a site corresponding to the $\mathrm{S} 3$ cleavage of Notch. EMBO Rep 2:835-841.

Schubert W, Prior R, Weidemann A, Dircksen H, Multhaup G, Masters CL, Beyreuther K (1991) Localization of Alzheimer beta A4 amyloid precursor protein at central and peripheral synaptic sites. Brain Res 563:184-194.

Selkoe D, Kopan R (2003) Notch and presenilin: regulated intramembrane proteolysis links development and degeneration. Annu Rev Neurosci 26:565-597.

Selkoe DJ (1991) The molecular pathology of Alzheimer's disease. Neuron 6:487-498.

Standen CL, Brownlees J, Grierson AJ, Kesavapany S, Lau KF, McLoughlin DM, Miller CC (2001) Phosphorylation of thr(668) in the cytoplasmic domain of the Alzheimer's disease amyloid precursor protein by stressactivated protein kinase $1 \mathrm{~b}$ (Jun N-terminal kinase-3). J Neurochem 76:316-320.

Sudo H, Jiang H, Yasukawa T, Hashimoto Y, Niikura T, Kawasumi M, Matsuda S, Takeuchi Y, Aiso S, Matsuoka M, Murayama Y, Nishimoto I (2000) Antibody-regulated neurotoxic function of cell-surface betaamyloid precursor protein. Mol Cell Neurosci 16:708-723.

Thinakaran G, Harris CL, Ratovitski T, Davenport F, Slunt HH, Price DL, Borchelt DR, Sisodia SS (1997) Evidence that levels of presenilins (PS1 and PS2) are coordinately regulated by competition for limiting cellular factors. J Biol Chem 272:28415-28422.

Torroja L, Luo L, White K (1996) APPL, the Drosophila member of the APP-family, exhibits differential trafficking and processing in CNS neurons. J Neurosci 16:4638-4650.

Torroja L, Packard M, Gorczyca M, White K, Budnik V (1999) The Drosophila $\beta$-amyloid precursor protein homolog promotes synapse differentiation at the neuromuscular junction. J Neurosci 19:7793-7803.

Vassar R, Bennett BD, Babu-Khan S, Kahn S, Mendiaz EA, Denis P, Teplow DB, Ross S, Amarante P, Loeloff R, Luo Y, Fisher S, Fuller J, Edenson S, Lile J, Jarosinski MA, Biere AL, Curran E, Burgess T, et al. (1999) betaSecretase cleavage of Alzheimer's amyloid precursor protein by the transmembrane aspartic protease BACE. Science 286:735-741.

Waetzig V, Herdegen T (2003) A single c-Jun N-terminal kinase isoform (JNK3-p54) is an effector in both neuronal differentiation and cell death. J Biol Chem 278:567-572.

Walsh DM, Fadeeva JV, LaVoie MJ, Paliga K, Eggert S, Kimberly WT, Wasco W, Selkoe DJ (2003) gamma-Secretase cleavage and binding to FE65 regulate the nuclear translocation of the intracellular C-terminal domain (ICD) of the APP family of proteins. Biochemistry 42:6664-6673.

Yang DD, Kuan CY, Whitmarsh AJ, Rincon M, Zheng TS, Davis RJ, Rakic P, Flavell RA (1997) Absence of excitotoxicity-induced apoptosis in the hippocampus of mice lacking the Jnk3 gene. Nature 389:865-870. 